\title{
As reservas particulares de patrimônio natural são eficientes para conservação
}

\section{florestal no Estado de Rondônia?}

\author{
Are private natural heritage reserves efficient for forest conservation in the State of Rondônia? \\ ¿Son eficientes las reservas del patrimonio natural privado para la conservación de los bosques en \\ el Estado de Rondônia?
}

Recebido: 17/08/2021 | Revisado: 22/08/2021 | Aceito: 23/08/2021 | Publicado: 25/08/2021

\author{
Micheli Leite Zanchetta \\ ORCID: https://orcid.org/0000-0002-5120-3444 \\ Universidade Federal de Rondônia, Brasil \\ E-mail: micheli_leite@hotmail.com \\ Diogo Martins Rosa \\ ORCID: https://orcid.org/0000-0003-3427-8353 \\ Secretaria de Estado do Desenvolvimento Ambiental, Brasil \\ E-mail: mrosa.diogo@gmail.com \\ Jhony Vendruscolo \\ ORCID: https://orcid.org/0000-0003-3043-0581 \\ Universidade Federal do Amazonas, Brasil \\ E-mail: jhonyvendruscolo@gmail.com \\ Wanderson Cleiton Schmidt Cavalheiro \\ ORCID: https://orcid.org/0000-0003-1356-8511 \\ Cavalheiro Engenharia Rural e Empresarial Ltda, Brasil \\ E-mail: engflo.ro@gmail.com \\ Karen Janones da Rocha \\ ORCID: https://orcid.org/0000-0002-2165-3081 \\ Universidade Feral de Rondônia, Brasil \\ E-mail: karenrocha@unir.br
}

\begin{abstract}
Resumo
O desmatamento na região amazônica vem crescendo muito nas últimas décadas, inclusive ilegalmente em Unidades de Conservação (UCs). E com intuito de reduzir o desmatamento, a legislação brasileira permite a criação de Reserva Particular de Patrimônio Natural (RPPN). Em face do exposto, o presente trabalho objetivou avaliar a eficiência das RPPNs para a conservação da cobertura florestal no Estado de Rondônia, Amazônia Ocidental. Foram realizados os monitoramentos de nove RPPNs, com análises temporais da cobertura do solo utilizando imagens dos satélites Landsat 5 (sensor TM) e Landsat 8 (sensor OLI), referentes aos anos de criação de cada RPPN e ao ano atual (2018), e classificação supervisionada (técnica Máxima Verossimilhança). Para realizar a classificação, foram coletas de 50 a 60 pixels por classe de interesse (água, solo exposto, floresta e perímetro urbano) com uso do Training Sample Manager. Com base no monitoramento foi possível observar o aumento de $\approx 54 \%$ de cobertura florestal na maioria das RPPNs do Estado, e detectar a eficiência dessas áreas na regeneração e preservação da vegetação nativa. Entretanto, a eficiência das RPPNs ocorre principalmente em zonas rurais, visto que as zonas urbanas tendem a se expandir, ocupando inclusive parte das áreas das RPPNs, como observado no município de Pimenta Bueno. Portanto, conclui-se que as RPPNs são eficientes para controlar o desmatamento em zonas rurais, porém são necessárias mais informações para auxiliar o planejamento de estratégias visando o aumento da eficiência destas Unidades de Conservação, principalmente em áreas próximas ou dentro de zonas urbanas.
\end{abstract}

Palavras-chave: Monitoramento; Uso sustentável; Políticas públicas.

\begin{abstract}
Deforestation in the Amazon region has been growing a lot in recent decades, including illegally in Conservation Units (CUs). And in order to reduce deforestation, Brazilian legislation allows the creation of a Private Natural Heritage Reserve (RPPN). In light of the above, this study aimed to evaluate the efficiency of RPPNs for the conservation of forest cover in the State of Rondônia, Western Amazon. Nine RPPNs were monitored, with temporal analyzes of land cover using images from the Landsat 5 (TM sensor) and Landsat 8 (OLI sensor) satellites, referring to the years of creation of each RPPN and the current year (2018), and supervised classification (Maximum Likelihood technique). To carry out the classification, 50 to 60 pixels were collected per class of interest (water, exposed soil, forest and urban perimeter) using the Training Sample Manager. Based on the monitoring, it was possible to observe an increase of $\approx 54 \%$ in forest cover in most of the RPPNs in the state, and to detect the efficiency of these areas in the regeneration and
\end{abstract}


preservation of native vegetation. However, the efficiency of RPPNs occurs mainly in rural areas, since urban areas tend to expand, even occupying part of the RPPNs areas, as observed in the municipality of Pimenta Bueno. Therefore, it is concluded that the RPPNs are efficient to control deforestation in rural areas, but more information is needed to assist the planning of strategies aimed at increasing the efficiency of these Conservation Units, especially in areas close to or within urban areas.

Keywords: Monitoring; Sustainable use; Public policy.

\section{Resumen}

La deforestación en la región amazónica ha crecido mucho en las últimas décadas, incluso ilegalmente en Unidades de Conservación (UC). Y para reducir la deforestación, la legislación brasileña permite la creación de una Reserva de Patrimonio Natural Privado (RPPN). A la luz de lo anterior, este estudio tuvo como objetivo evaluar la eficiencia de las RPPN para la conservación de la cubierta forestal en el Estado de Rondônia, Amazonia Occidental. Se monitorearon nueve RPPN, con análisis temporales de cobertura terrestre utilizando imágenes de los satélites Landsat 5 (sensor TM) y Landsat 8 (sensor OLI), referentes a los años de creación de cada RPPN y el año actual (2018), y clasificación supervisada. (Técnica de máxima verosimilitud). Para realizar la clasificación se recolectaron de 50 a 60 píxeles por clase de interés (água, suelo expuesto, bosque y perímetro urbano) utilizando el Training Sample Manager. Con base en el monitoreo, fue posible observar el aumento de $\approx 54 \%$ en la cobertura forestal en la mayoría de las RPPN del estado, y detectar la eficiencia de estas áreas en la regeneración y preservación de la vegetación nativa. Sin embargo, la eficiencia de las RPPN se dá principalmente en las áreas rurales, ya que las áreas urbanas tienden a expandirse, incluso ocupando parte de las áreas de las RPPN, como se observa en el municipio de Pimenta Bueno. Por lo tanto, se concluye que las RPPN son eficientes para controlar la deforestación en áreas rurales, pero se necesita más información para ayudar en la planificación de estrategias dirigidas a incrementar la eficiencia de estas Unidades de Conservación, especialmente en áreas cercanas o dentro de áreas urbanas.

Palabras clave: Monitoreo; Uso sustentable; Políticas públicas.

\section{Introdução}

A redução de áreas de florestas nativas em todo o mundo vem ocorrendo como resultado não só de fenômenos naturais, como também pela ação humana com cortes de árvores para fins comerciais e exploração irregular, expansão de terras para utilização agropecuária e a ocorrência de incêndios florestais (Arraes et al., 2012; Freitas et al., 2018; Sathler et al., 2018). Na Amazônia brasileira, o desmatamento se acelerou de forma significativa durante os anos de 1990 e início dos anos 2000 , atingindo uma taxa anual de $27.423 \mathrm{~km} 2$ em 2004, porém, no ano de 2017 essa taxa teve uma redução de $75 \%$ (7.989 km2) (INPE, 2017). Essas taxas foram, principalmente, promovidas pela expansão de infraestruturas e novas atividades econômicas (Fearnside \& Graça, 2009).

As grandes mudanças na demografia regional, na urbanização e nos efeitos ecológicos causados pelo desmatamento e degradação florestal, afetam significativamente o sistema climático global, a agricultura e os serviços ecológicos existentes na Amazônia brasileira (Soares-Filho et al., 2004; Soares-Filho et al., 2005; Fearnside, 2006). Neste contexto, constata-se a importância das Unidades de Conservação (UCs) como estratégia para o controle do desmatamento e, consequentemente, a conservação da biodiversidade e da cobertura florestal a nível mundial (Marques \& Nucci, 2007). As UCs exercem uma série de serviços ambientais, como a regulação do clima, o ciclo da água e do carbono, a conservação do meio ambiente, turismo, incentivo à educação ambiental e segurança alimentar, cujos benefícios são usufruídos por grande parte da população brasileira, inclusive por setores econômicos em contínuo crescimento (Nogueira et al., 2018).

No Brasil, o Sistema Nacional de Unidades de Conservação (SNUC), regulamentado pela Lei no 9.985 , de 18 de julho de 2000, é quem determina os critérios e normas para a criação, implantação e gestão das UCs, sendo estas divididas em duas categorias: proteção integral e uso sustentável (Brasil, 2000). O Estado de Rondônia possui o Sistema Estadual de Unidades de Conservação de Rondônia (SEUC/RO), constituído pelo conjunto de UCs localizadas dentro do Território, que visa à manutenção da diversidade biológica e dos recursos genéticos no território estadual e águas jurisdicionais (Rondônia, 2002). As UCs são geridas pelo poder público, com exceção para Reserva Particular do Patrimônio Natural (RPPN), considerada de iniciativa privada (Brasil, 2000).

As RPPNs foram criadas para conservar e proteger terras privadas, prevista desde o antigo Código Florestal (Brasil, 
1965), e na Política Agrícola (Brasil, 1991). Entretanto, por meio do decreto no 1.922, de 5 de junho de 1996 (Brasil, 1996), as RPPNs foram definidas como uma categoria de UC, sendo, então, legitimadas pelo Instituto Brasileiro do Meio Ambiente e dos Recursos Naturais Renováveis (IBAMA) e, atualmente pelo Instituto Chico Mendes de Conservação da Biodiversidade (ICMbio). Assim, são definidas como áreas de domínio privado a serem especialmente protegidas por iniciativa de seu proprietário.

As áreas localizadas nas RPPNs possuem importante papel para o desenvolvimento de atividades de cunho científico, cultural, educacional, recreativo e de lazer (BRASIL, 2006). Deste modo, fazem-se necessários estudos e ferramentas para a implantação de projetos voltados para o desenvolvimento local, assim como a necessidade de envolvera sociedade para intensificar as ações de monitoramento e proteger os recursos naturais.

Como ferramenta para o monitoramento é de grande importância nesse cenário de desmatamento em UCs, o uso de geotecnologias pode ser aplicado diretamente para elaboração e/ou enriquecimento de bancos de dados georreferenciados, utilizados para identificar regiões críticas, e, portanto, auxiliar o planejamento de estratégias para recuperação, conservação ou preservação das UCs (Nascimento et al., 2009).Assim, essa pesquisa tem por objetivo analisar a eficiência das RPPNs para conservação florestal no Estado de Rondônia, com base em monitoramentos da cobertura do solo com imagens de satélite.

\section{Material e Métodos}

\subsection{Caracterização e localização das áreas de estudo}

O estudo foi realizado em nove RPPNs, localizadas em seis municípios do Estado de Rondônia (Figura 1). As RPPNs foram criadas legalmente ao longo de um período de 15 anos, de 1997 a 2012 (Tabela 1). A sobreposição dos polígonos das RPPNs 7 e 8 ocorreu por serem criadas antes do ano de 2006, quando não era obrigatório a entrega de georreferenciamento da área (Brasil, 2006), mas pelo decreto de criação e tamanho da área, as duas ficam em locais diferentes.

Figura 1. Mapa de localização das Reservas Particulares de Patrimônio Natural (RPPNs) no Estado de Rondônia.

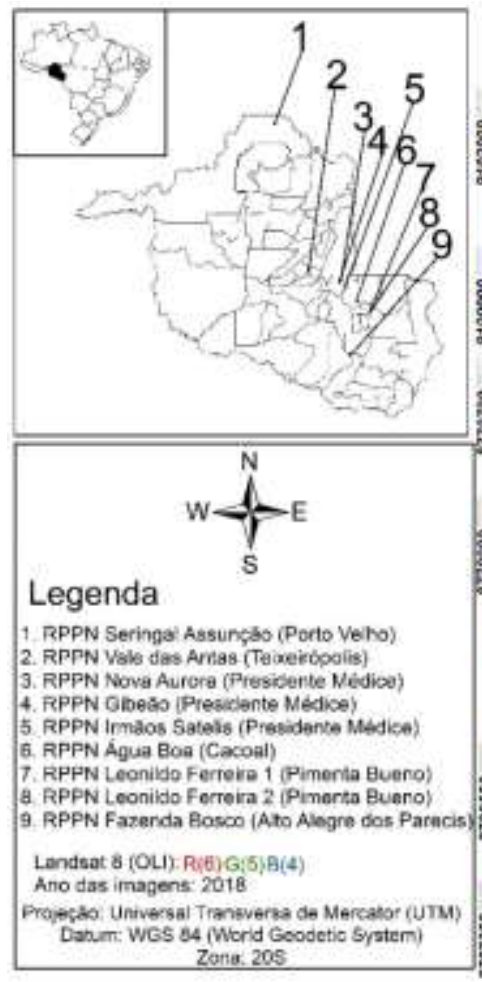
Zane. 205
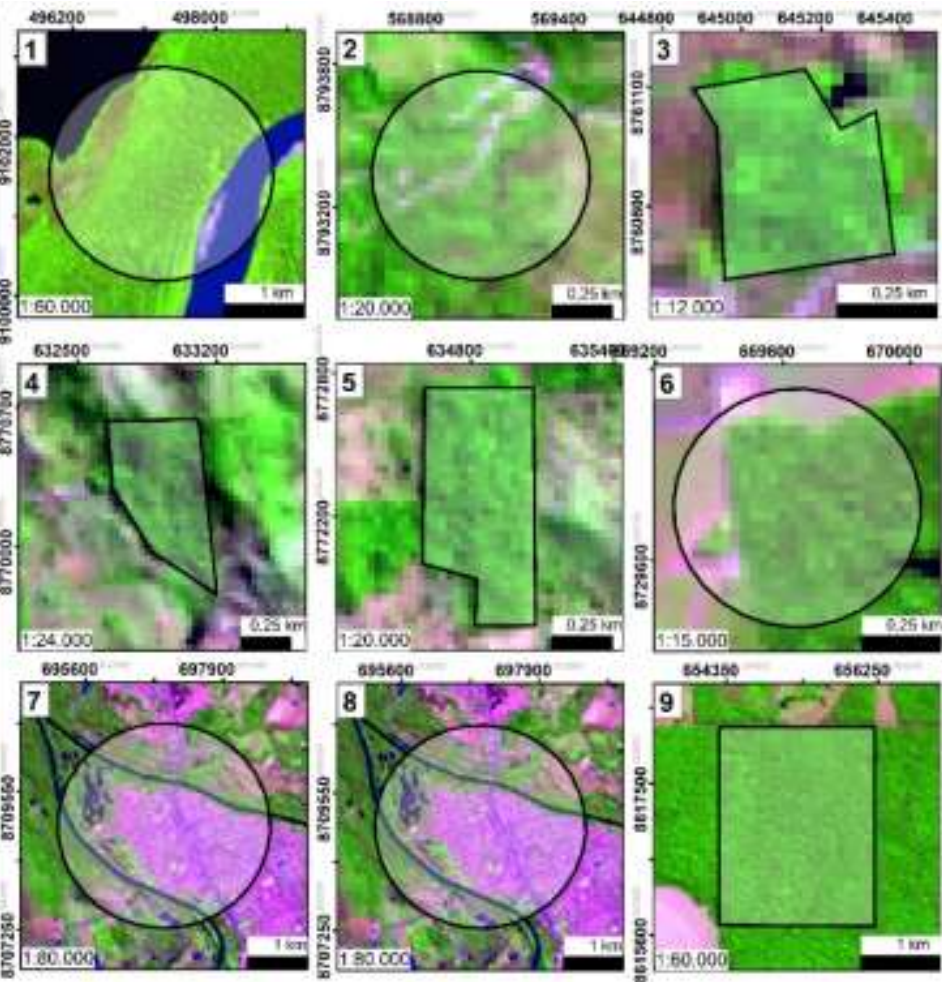

Fonte: Autores (2021). 
Tabela 1. Informações sobre as Reservas Particulares de Patrimônio Natural (RPPNs) do Estado de Rondônia.

\begin{tabular}{ccccc}
\hline $\mathbf{N}^{\circ}$ & RPPN & Município & Área (ha) & $\begin{array}{c}\text { Portaria } \\
(\mathbf{n} \% \mathbf{a n o})\end{array}$ \\
\hline 1 & Seringal Assunção & Porto Velho & 589,96 & $63 / 1997$ \\
2 & Vale das Antas & Teixeirópolis & 62,42 & $81-\mathrm{N} / 1999$ \\
3 & Nova Aurora & Presidente Médici & 18,53 & $110 / 2011$ \\
4 & Gibeão & Presidente Médici & 31,30 & $68 / 2012$ \\
5 & Irmãos Satelis & Presidente Médici & 45,41 & $122 / 2012$ \\
6 & Água Boa & Cacoal & 45,41 & $21 / 2000$ \\
7 & Leonildo Ferreira 1 & Pimenta Bueno & 952,54 & $173 / 2001$ \\
8 & Leonildo Ferreira 2 & Pimenta Bueno & 938,86 & $175 / 2001$ \\
9 & Fazenda Bosco & Alto Alegre dos Parecis & 486,72 & $32 / 2005$ \\
\hline
\end{tabular}

Fonte: SimRPPN (2018)

No geral, o Estado possui clima do tipo Monção (Am) (Alvares et al., 2013), com precipitação anual média de 1.728,9 a 2.243,8 mm ano ${ }^{-1}$, máxima nos meses de janeiro a março (Franca, 2015); umidade relativa anual média de 75\%, temperatura anual média de $25,9^{\circ} \mathrm{C}$ (Sedam, 2012) e vegetação nativa predominante classificada como Floresta Ombrófila Aberta (IBGE, 2012).

\subsection{Caracterização e monitoramento da cobertura do solo}

Para caracterização da cobertura do solo, utilizou-se imagens do satélite Landsat 5, sensor TM (Thematic Mapper), correspondentes aos anos de criação das RPPNs; e do Landsat 8, sensor OLI (Operational Land Imager), para o ano de 2018, utilizando-se da composição colorida (RGB) verdadeira (L5: 3/2/1; L8: 4/3/2) e falsa cor (L5: 5/4/3; L8: 6/5/4) para melhor visualização e qualidade das imagens.

As imagens foram adquiridas no site da United States Geological Survey da coleção Level 2 (USGS, 2018), e correspondem aos meses de junho a agosto, por terem menores incidências de nuvens, e para manter a angulação de azimute e elevação solar (Tabela 2). Utilizou-se também as imagens de alta resolução do software Google Earth Pro para verificar situação das RPPNs de forma mais nítida.

Tabela 2. Informações das imagens de satélite utilizadas para o monitoramento das Reservas Particulares de Patrimônio Natural (RPPNs) do Estado de Rondônia.

\begin{tabular}{|c|c|c|c|c|c|c|c|c|c|}
\hline \multirow{3}{*}{$\mathrm{N}^{\circ}$} & \multirow{3}{*}{ RPPN } & \multicolumn{8}{|c|}{ Satélite } \\
\hline & & \multicolumn{4}{|c|}{ Landsat 5} & \multicolumn{4}{|c|}{ Landsat 8} \\
\hline & & Ano & $\begin{array}{l}\text { Resolução } \\
\text { espacial }\end{array}$ & $\begin{array}{l}\text { Azimute } \\
\text { Solar }\end{array}$ & $\begin{array}{l}\text { Elevação } \\
\text { Solar }\end{array}$ & Ano & $\begin{array}{l}\text { Resolução } \\
\text { espacial }\end{array}$ & $\begin{array}{l}\text { Azimute } \\
\text { Solar }\end{array}$ & $\begin{array}{l}\text { Elevação } \\
\text { Solar }\end{array}$ \\
\hline & & & --- $\mathrm{m}$--- & --- ${ }^{\circ}---$ & --- ${ }^{o}---$ & & $---\mathrm{m}---$ & $--{ }^{0}---$ & --- ${ }^{\circ}---$ \\
\hline 1 & Seringal Assunção & 1997 & 30 & 47,4 & 42,1 & 2018 & 30 & 42,5 & 47,4 \\
\hline 2 & Vale das Antas & 1999 & 30 & 49,8 & 43,4 & 2018 & 30 & 43,3 & 47,0 \\
\hline 3 & Nova Aurora & 2011 & 30 & 52,4 & 48,8 & 2018 & 30 & 42,5 & 45,8 \\
\hline 4 & Gibeão & $2011 *$ & 30 & 52,4 & 48,8 & 2018 & 30 & 42,5 & 45,8 \\
\hline 5 & Irmãos Satelis & $2011 *$ & 30 & 52,4 & 48,8 & 2018 & 30 & 42,5 & 45,8 \\
\hline 6 & Água Boa & 2000 & 30 & 44,3 & 41,0 & 2018 & 30 & 39,4 & 44,7 \\
\hline 7 & Leonildo Ferreira 1 & 2001 & 30 & 43,4 & 42,5 & 2018 & 30 & 39,4 & 44,7 \\
\hline 8 & Leonildo Ferreira 2 & 2001 & 30 & 43,4 & 42,5 & 2018 & 30 & 39,4 & 44,7 \\
\hline 9 & Fazenda Bosco & 2005 & 30 & 45,3 & 43,2 & 2018 & 30 & 43,5 & 45,8 \\
\hline
\end{tabular}

*As imagens foram adquiridas no ano de 2011, visto que no ano de criação das RPPNs as imagens estavam sem qualidade. Fonte: Autores (2021).

Para o processamento das imagens, utilizou-se o software ArcGIS 10.5, com a RGB - falsa cor (L5: R5, G4, B3 e L8: R6, G5, B4), a qual é composta por bandas de Infravermelho Médio (SWIR), Infravermelho Próximo (NIR) e Vermelho (RED). 
Após a composição da falsa cor, foram coletadas amostras de 50 a 60 pixels com uso do Train Sample Manager (TSM), para cada classe de interesse (água, solo exposto, floresta e perímetro urbano), por meio de ROIs, em formatos de retângulos e círculos. Em seguida, verificou-se as separações espectrais das três classes: água, floresta e solo exposto para todas as RPPNs. Para as RPPNs localizadas no município de Pimenta Bueno foi acrescentada a classe de perímetro urbano.

Posteriormente, com as amostras espectrais, utilizou-se a classificação supervisionada com a função Maximum Likelihood Classification (Máxima Verossimilhança), um classificador "pixel a pixel" que utiliza de forma individual a informação espectral de cada pixel, na busca por regiões homogêneas, e em seguida, convertidos em polígonos. Feita a transformação da imagem (raster) para polígono (vector), utilizou-se da ferramenta "editor" para algumas correções em relação à classificação dos pixels. Por fim, recortou (clip) a imagem com base no limite da RPPN. O cálculo das classes foi realizado por meio da área total de cada RPPN, apresentados em porcentagem (\%), e por diferença entre as mesmas para os anos de criação e atual (2018).

\section{Resultados}

Com o monitoramento das RPPNs, foi possível observar mudanças na cobertura do solo do ano de criação até 2018 (Figuras 2 a 8). Na RPPN Seringal Assunção, observa-se que parte da água que havia no local quando a mesma foi criada desapareceu, dando espaço para formação de bancos de areia em seu lugar, classificadas como solo exposto. Nessa RPPN, em 21 anos após sua criação, o aumento em floresta foi de 12,27 ha (2,08\%), o aumento em solo exposto foi de 64,35 ha (10,91\%) e a redução em água foi de 76,61 ha (12,99\%) (Figura 2 e 3).

Na RPPN Vale das Antas, o cenário é totalmente diferente, a mesma demonstrou um aumento de floresta de 21,66 ha (34,60\%) e uma redução em solo exposto de 21,58 ha (72,91\%), o que caracteriza boa recuperação da área, visto que 64,35\% de solo exposto já foram restaurados por meio da conservação da cobertura florestal. Na RPPN Água Boa não foi observado o mesmo potencial de recuperação florestal quando comparada a RPPN Vale das Antas. Para essa RPPN, verificou-se somente o aumento em floresta de 1,51 ha $(3,21 \%)$, redução me solo exposto de 1,26 ha $(2,81 \%)$ e aumento em água de 0,18 ha $(0,40 \%)$ (Figura 2 e 3). 
Figura 2. Cobertura do solo nas Reservas Particulares de Patrimônio Natural (RPPNs) Seringal Assunção, Vale das Antas e Água Boa, nos respectivos anos de criação e no ano de 2018.
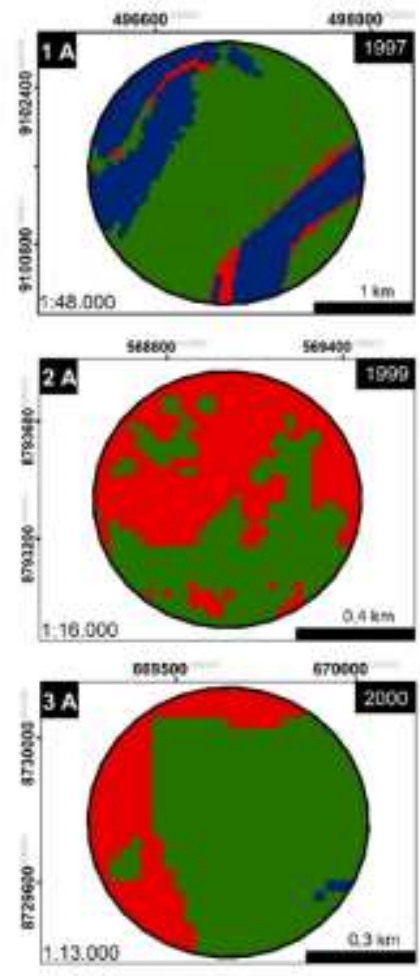
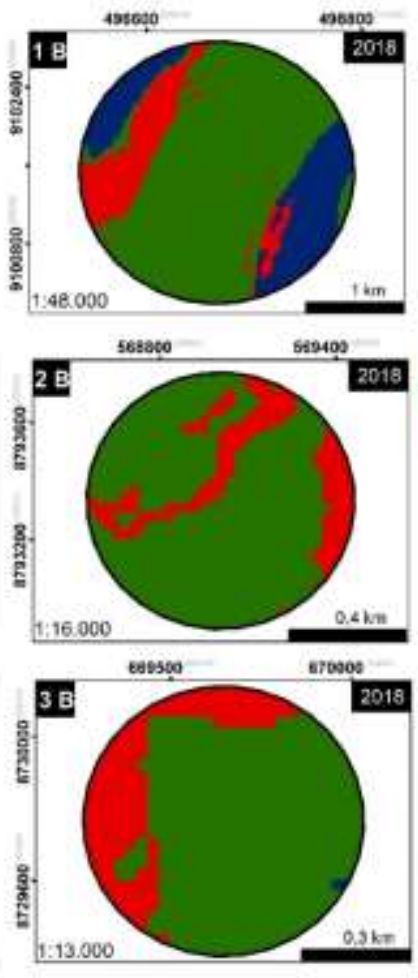

Fonte: Autores (2021).

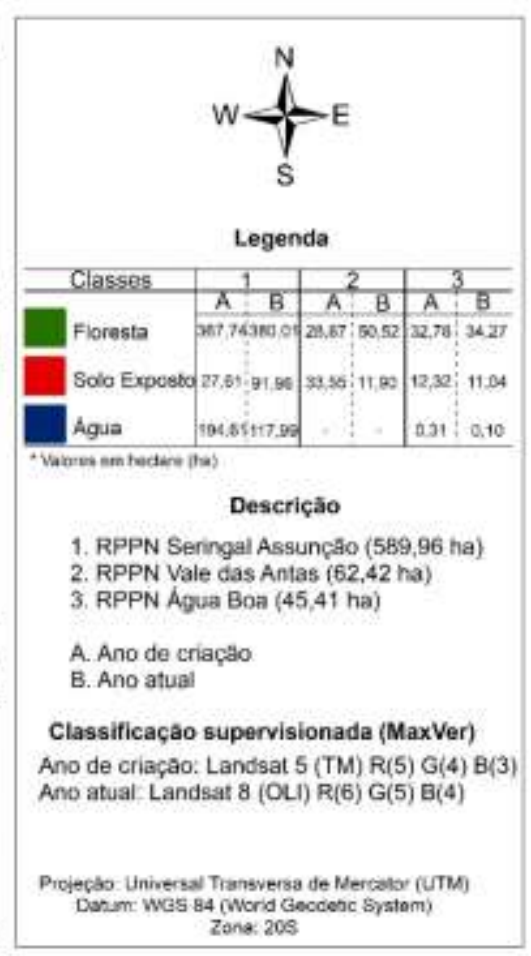

Donum Was 84 imord Geociesc Syatam) Zona: 208

Figura 3. Percentual de água, solo exposto e floresta nas Reservas Particulares de Patrimônio Natural (RPPNs) Seringal Assunção, Vale das Antas e Água boa, nos respectivos anos de criação e no ano de 2018.

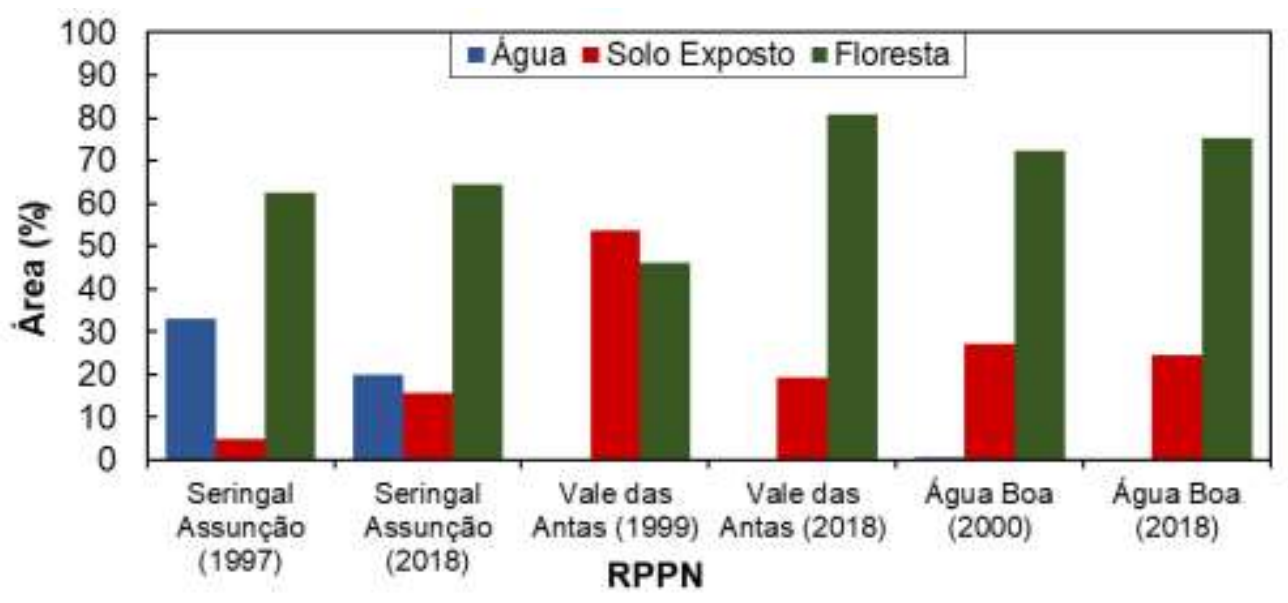

Fonte: Autores (2021).

As RPPNs Leonildo Ferreira 1 (995,48 ha) e Leonildo Ferreira 2 (981,18 ha), ambas do município de Pimenta Bueno, pertencem ao mesmo proprietário e possuem diferença de 14,3 ha em área total. Nessas RPPNs, houve aumento significativo no seu perímetro urbano, explicado pelo aumento da população no município de Pimenta Bueno, assim como a redução significativa das classes observadas. No ano de 2001, a população do município era de 31.701 pessoas, enquanto que no ano de 2018 se estima que a população esteja com 36.434 pessoas (IBGE, 2018). Sendo assim, o perímetro urbano teve um aumento de $\approx 167$ ha (18\%) nesses 17 anos de criação, enquanto a área de floresta reduziu $\approx 100$ ha $(10 \%)$; o que pode ter corroborado com o aumento de $\approx$ 52 ha $(5,5 \%)$ de corpos de água mais visíveis atualmente, e a redução de $\approx 119$ ha $(12,50 \%)$ de solo exposto (Figura 4 e 5 ). 
Na RPPN Fazenda Bosco, observa-se que em 13 anos ocorreu aumento da área de floresta de 16,02 ha (3,29\%) e, desta forma, a área de solo exposto atual é de apenas 0,09 ha (0,02\%) (Figura 4 e 5). Assim, nessa RPPN não se visualizou intervenção alguma, e os resultados demonstram que a mesma está de acordo com essa pesquisa.

Figura 4. Cobertura do solo nas Reservas Particulares de Patrimônio Natural (RPPNs) Leonildo Ferreira 1, Leonildo Ferreira 2 e Fazenda Bosco, nos respectivos anos de criação e no ano de 2018.
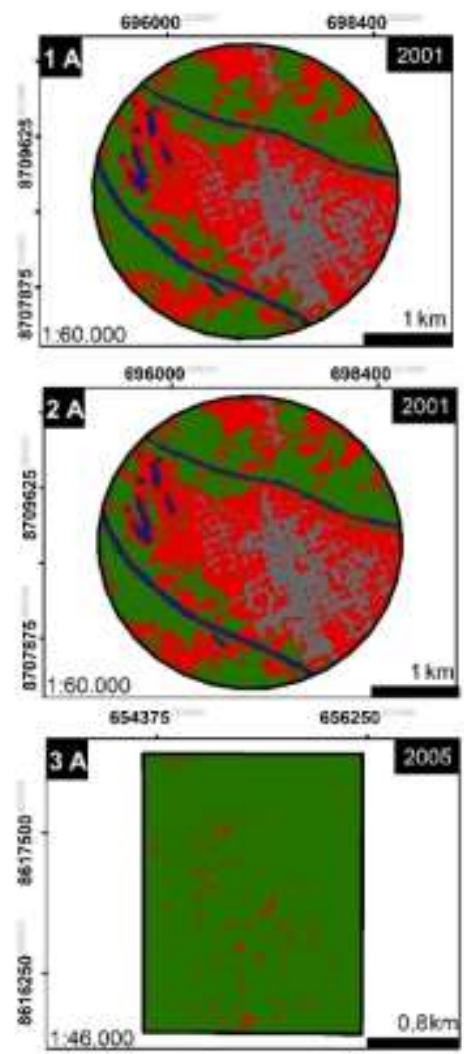
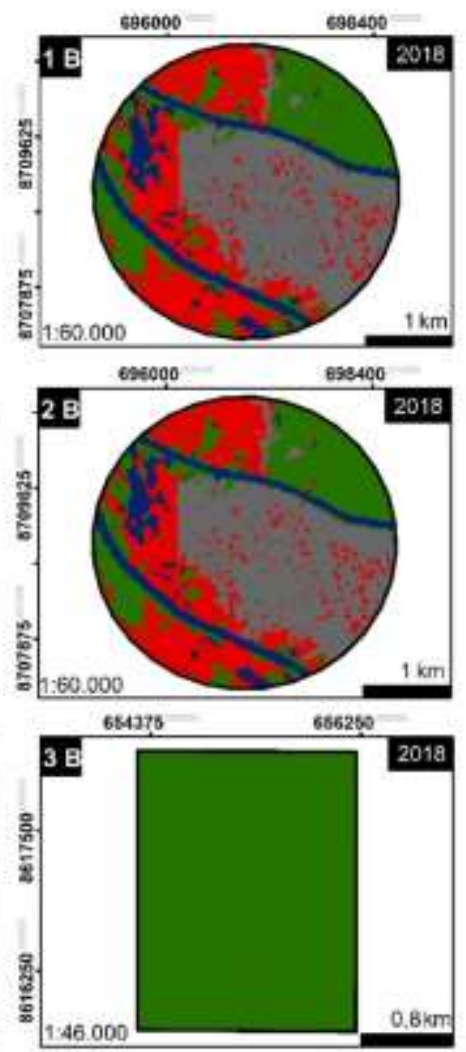

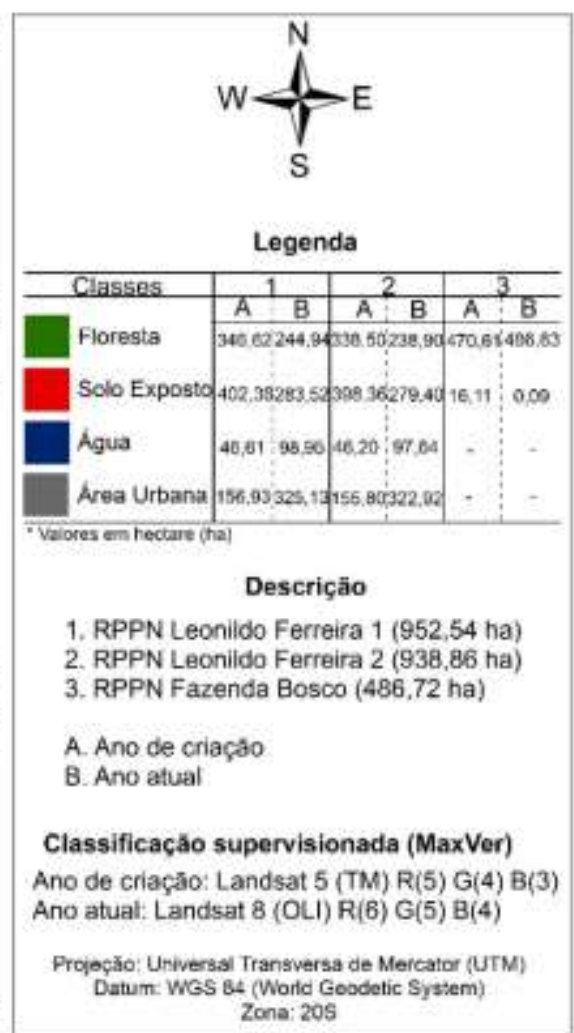

Fonte: Autores (2021). 
Figura 5. Percentual de água, solo exposto, floresta e perímetro urbano do monitoramento nas Reservas Particulares de Patrimônio Natural (RPPNs) Leonildo Ferreira 1, Leonildo Ferreira 2 e Fazenda Bosco, nos respectivos anos de criação e no ano de 2018.

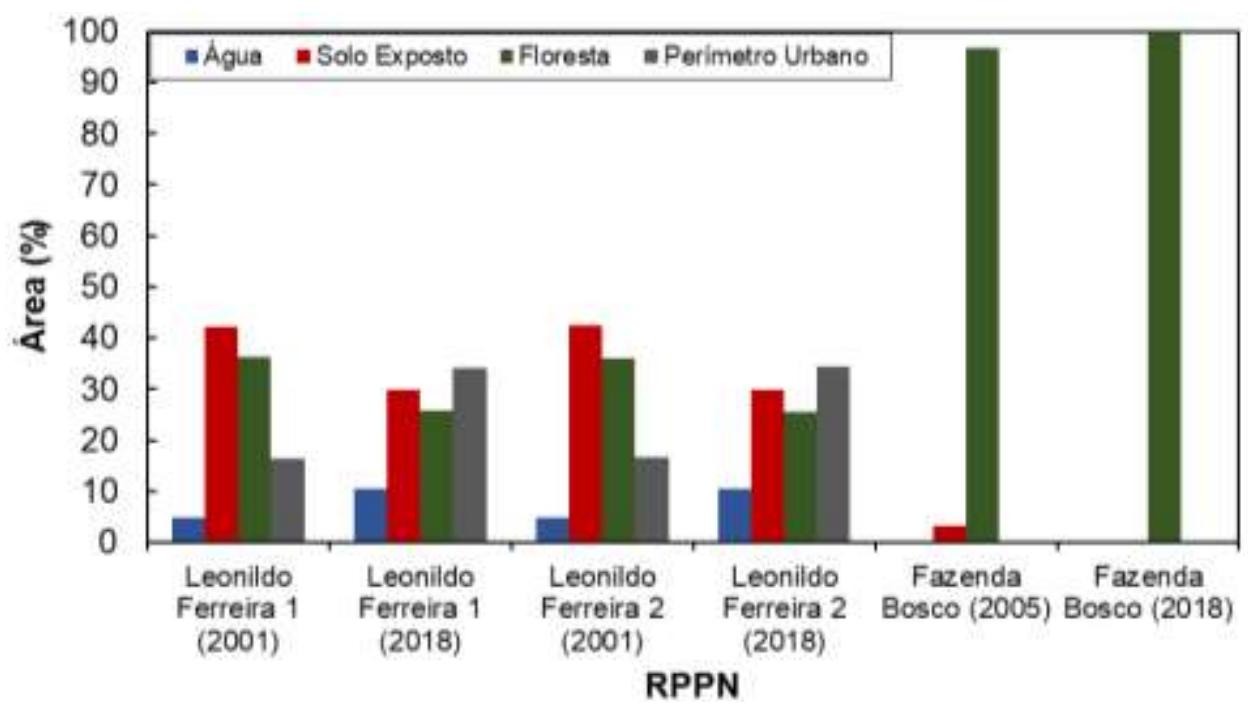

Fonte: Autores (2021).

Para os resultados encontrados nas RPPNs de Pimenta Bueno, foram observados um padrão diferente do encontrado nas demais RPPNs estudadas em Rondônia. As RPPNs de Pimenta Bueno estão em perímetro urbano, assim as mesmas deveriam impedir a expansão urbana sobre áreas naturais e/ou florestadas, porém constata-se um cenário totalmente oposto. Contudo, a expansão urbana poderia ter sido maior se não existisse essas RPPNs e por isso são necessários mais estudos em relação às RPPNs que estão localizadas próximas e em perímetros urbanos.

Entre os anos de 2011 e 2012, foram criadas três RPPNs no município de Presidente Médici, as RPPNs: (i) Nova Aurora, (ii) Gibeão e (iii) Irmãos Satelis. Todas obtiveram aumento de vegetação e redução de solo exposto. Nos seis e sete anos observados, a regeneração foi considerada rápida quando comparadas com as demais RPPNs do estado. Na RPPN Nova Aurora, foi observado um aumento em área de floresta de 0,50 ha $(2,67 \%)$, assim como uma redução proporcional da área de solo exposto. Nas RPPNs Gibeão e Irmãos Satelis, verificaram-se aumentos nas áreas de floresta, 0,48 ha (1,53\%) e 2,38 ha (5,78\%) respectivamente, e reduções das áreas de solo exposto, também nas mesmas proporções (Figuras 6 e 7). 
Figura 6. Cobertura do solo para o monitoramento nas Reservas Particulares de Patrimônio Natural (RPPNs) Nova Aurora, Gibeão e Irmãos Satelis, nos respectivos anos de criação e no ano de 2018.
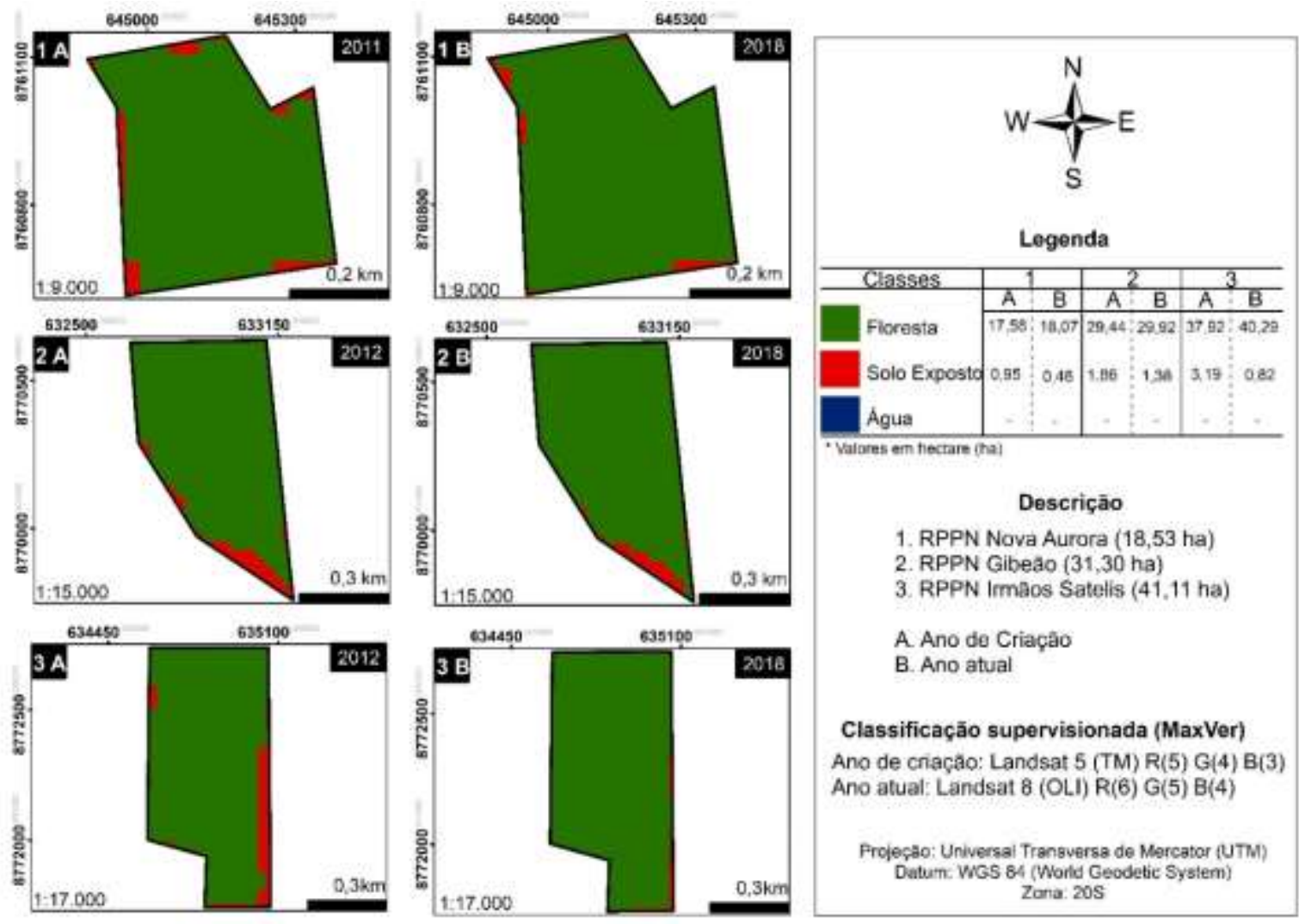

Fonte: Autores (2021).

Figura 7. Percentual de solo exposto e floresta do monitoramento nas Reservas Particulares de Patrimônio Natural (RPPNs) Nova Aurora, Gibeão e Irmãos Satelis, nos respectivos anos de criação e no ano de 2018.

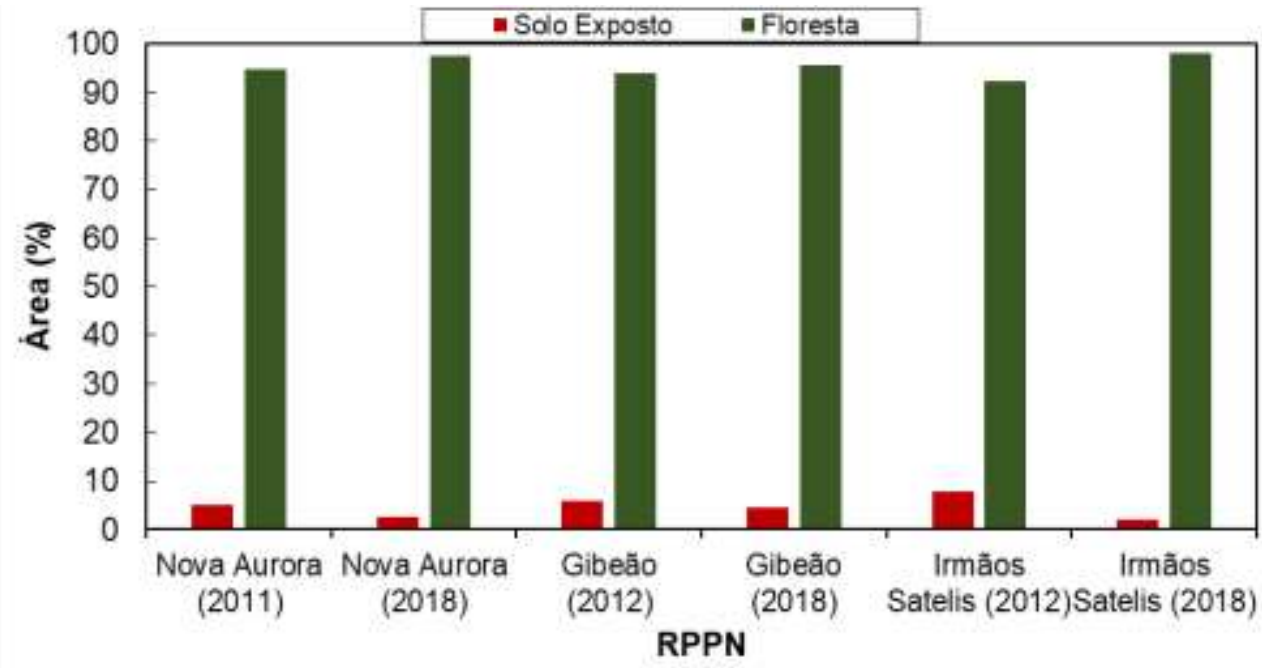

Fonte: Autores (2021).

\section{Discussão}

No monitoramento das RPPNs Seringal Assunção, Vale das Antas e Água Boa, observa-se a eficiência quando o assunto é conservação. As mesmas comprovam que quando uma área é protegida antes de perder toda sua cobertura vegetal, tem-se o 
potencial de regeneração natural aumentado (Almeida \& Sánchez, 2005). Essa regeneração é conhecida como uma sucessão secundária, podendo ser avaliada mediante o uso de indicadores de desempenho, verificando a real eficiência da cobertura vegetal na proteção dos solos (Reys et al., 2005). Na RPPN Seringal Assunção, foram observadas áreas (pixels) sem vegetação no meio da floresta, após verificar seus tamanhos, os mesmos indicam que sejam possíveis clareiras causadas por distúrbios climáticos. Estas não são prejudiciais para a conservação e manutenção dos ecossistemas naturais, podendo as clareiras serem consideradas como um mecanismo de manutenção da biodiversidade (Hartshorn, 1980; Brokaw, 1982; Whitmore, 1988).

As RPPNs localizadas em zonas urbanas, como as RPPNs Leonildo Ferreira 1 e 2, são utilizadas como estratégias para impedir a expansão urbana sobre as áreas naturais e/ou florestadas (Brasil, 2000; PL N. ${ }^{\circ}$ 1.548-B, de 2015), porém, as mesmas não têm direito à isenção do IPTU (Imposto Predial e Territorial Urbano) (Souza et al., 2012). Como sugestão para incentivar a proteção e manutenção de áreas verdes pelos municípios, a isenção do IPTU poderia ser revista nos próximos projetos de Lei, por exemplo, nas alterações do Projeto Lei nº 1.548-B, de 2015 que está em andamento.

Com relação às RPPNs Nova Aurora, Gibeão e Irmãos Satelis, no período avaliado, 7 anos, foram observados que a cobertura florestal permaneceu conservada. Esse resultado também foi observado em UC e Estação Ecológica, do Cerrado no sudeste do Brasil (Pinheiro \& Durigan, 2009).

No geral, das noves RPPNs monitoradas, apenas três obtiveram resultados diferentes ao desejado de cobertura do solo exposto por floresta. A RPPNs Seringal Assunção obteve aumento de solo exposto com o surgimento de bancos de areia em áreas que antes eram brejosas, porém ainda foi observado aumento em floresta (Figura 8). As RPPNs Leonildo Ferreira 1 e 2 , pelo fato de as mesmas estarem dentro do perímetro urbano, acreditava-se que a expansão urbana fosse menor, em contrapartida, mesmo com o aumento do perímetro urbano as RPPNs podem ter auxiliado para que esse aumento não acontecesse de forma desenfreada, pois ainda há uma porcentagem significativa de vegetação nessas RPPNs. E apesar da criação das RPPNs, se não houver conscientização da população circunvizinha, a UC não será suficiente para garantir a conservação do fragmento florestal. Nas demais RPPNs, Vale das Antas, Água Boa, Nova Aurora, Gibeão, Irmãos Satelis e Fazenda Bosco, houve a redução de solo exposto e, consequentemente, aumento de sua área de floresta, confirmando que as RPPNs podem ser uma forma eficiente para a conservação da cobertura florestal (Figura 8). 
Figura 8. Evolução das RPPNs do Estado de Rondônia, em relação a ocupação do solo desde sua criação até o ano de 2018.

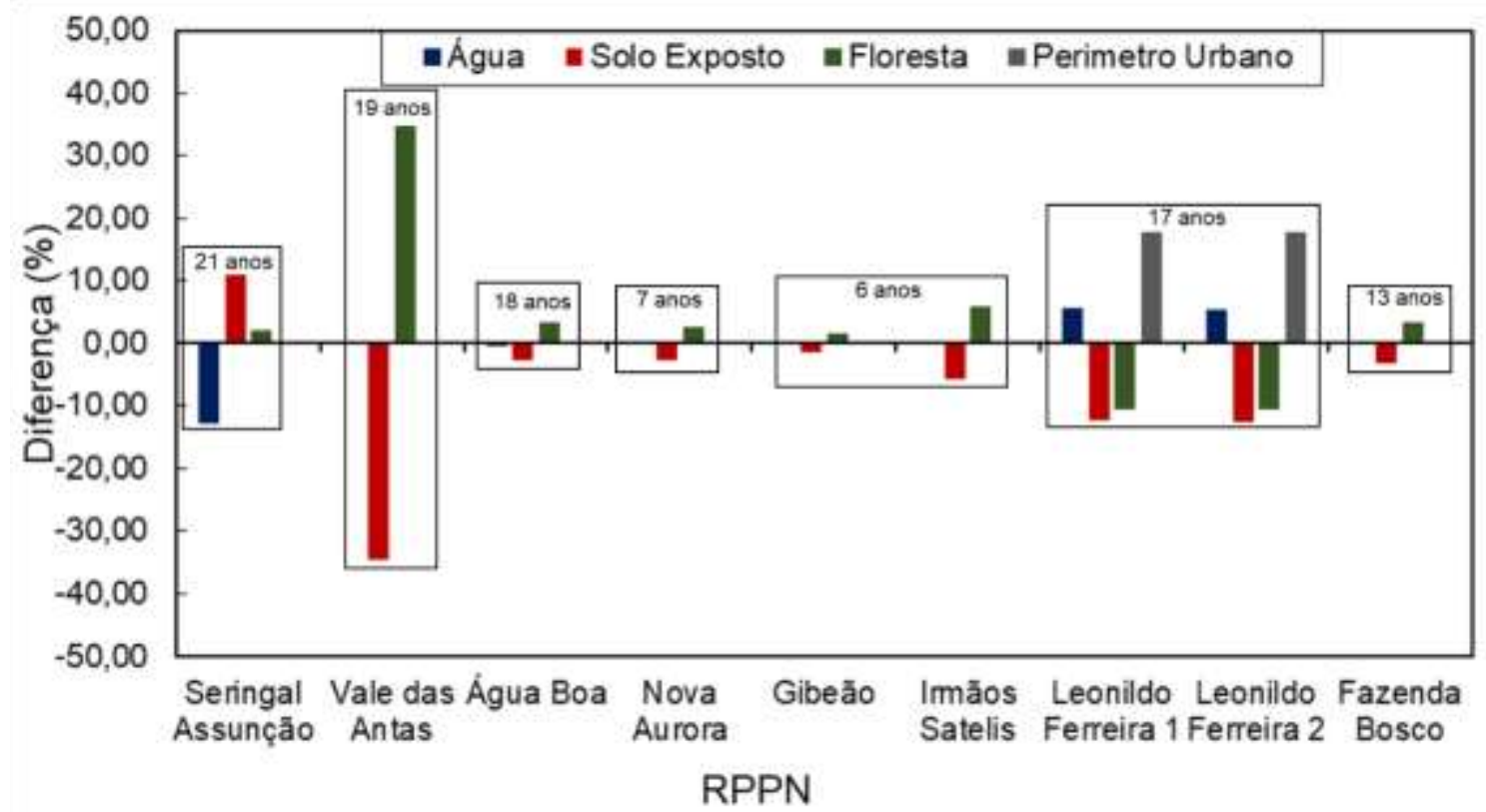

Fonte: Autores (2021).

Entre os anos de 2002 e 2011, foi verificado o aumento de 400\% do desmatamento ilegal dentro de áreas protegidas no Estado (Piontekowsk et al., 2014), porém sem a criação de estratégias adequadas, a tendência desse desmatamento ilegal é persistir. Tendo em vista o monitoramento realizado nas RPPNs do Estado até o ano de 2018, verifica que as mesmas podem ser uma alternativa para deter o desmatamento ilegal no Estado de Rondônia. Os remanescentes florestais sem a devida conservação e/ou proteção ficam sujeitos a sério risco de descaracterização e degradação, ou até mesmo de completa destruição. E as UCs, reforçando o potencial da RPPN, favorecem a regeneração das áreas degradadas; mas dependendo das condições atuais do remanescente florestal, somente a criação da UC não é suficiente.

Políticas de conscientização ambiental da população, principalmente no caso das UCs em perímetro urbano, e incentivos por parte do Estado e dos municípios para a criação e manutenção das RPPNs, assim como outras UCs, são necessários para que as UCs sejam eficientes. Ademais, faz-se necessário criação de estímulos para os proprietários preservarem suas áreas de floresta nativa, tanto por parte do Estado de Rondônia como também dos 52 municípios, principalmente dos 46 municípios sem RPPNs.

\section{Conclusão}

As RPPNs no Estadode Rondônia são uma solução eficiente para a preservação da vegetação nativa e para o controle do desmatamento. Porém, não são suficientes para conter a expansão urbana. Ainda são necessárias mais pesquisas nesse sentido, visto que não há monitoramento de UCs em outras regiões ou estados do Brasil, assim como as áreas no entorno das mesmas; e conscientização da população sobre a importância das mesmas.

\section{Referências}

Almeida, R. O. P. O., \& Sánchez, L. E. (2005). Revegetação de áreas de mineração: critérios de monitoramento e avaliação do desempenho. Revista Árvore, 29 (1): 47-54. https://doi.org/10.1590/S0100-67622005000100006

Alvares, C. A., Stape, J. L., Sentelhas, P. C., de Moraes, G., Leonardo, J., \& Sparovek, G. (2013). Köppen's climate classification map for Brazil. Meteorologische Zeitschrift, 22 (6): 711-728. https://doi.org/10.1127/0941-2948/2013/0507

Arraes, R. A., Mariano, F. Z., \& Simonassi, A. G. (2012). Causas do desmatamento no Brasil e seu ordenamento no contexto mundial. Revista de Economia e Sociologia Rural, 50 (1): 119-140. https://doi.org/10.1590/S0103-20032012000100007 
Brasil (1991). Lei no 8.171, de 17 de janeiro de 1991. Dispõe sobre a política agrícola. http://www.planalto.gov.br/ccivil_03/LEIS/L8171.htm

Brasil (1996). Decreto no 1.922, de 5 de junho de 1996. Dispõe sobre o reconhecimento das Reservas Particulares do Patrimônio Natural, e dá outras providências. http://www.planalto.gov.br/ccivil_03/decreto/Antigos/D1922.htm

Brasil (2000). Lei $\mathrm{n}^{\circ}$ 9.985, de 18 de julho de 2000. Institui o Sistema Nacional de Unidades de Conservação da Natureza e dá outras providências. http://www.planalto.gov.br/ccivil_03/LEIS/L9985.htm

Brasil (2006). Decreto $n^{\circ} 5.746$, de 5 de abril de 2006. Regulamenta o art. 21 da Lei no 9.985, de 18 de julho de 2000, que dispõe sobre o Sistema Nacional de Unidades de Conservação da Natureza. http://www.planalto.gov.br/ccivil_03/_Ato2004-2006/2006/Decreto/D5746.htm

Brokaw, N. V. L. (1982). The definition of treefall gap and its effect on measures of forest dynamics. Biotropica, 14 (2):158-160. https://doi.org/10.2307/2387750

Franca, R. R. (2015). Climatologia das chuvas em Rondônia - período 1981-2011. Revista Geografias, 1 (20): 44-58. https://periodicos.ufmg.br/index.php/geografias/article/view/13392

Fearnside, P. M., \& Graça, P. M. L. A. (2009). BR-319: A rodovia Manaus-Porto Velho e o impacto potencial de conectar o arco de desmatamento à Amazônia central. Novos cadernos NAEA, 12 (1): 19-50. https://doi.org/10.5801/ncn.v12i1.241

Fearnside, P. M (2006). Desmatamento na Amazônia: dinâmica, impactos e controle. Acta Amazônica, 36 (3): 395-400. https://doi.org/10.1590/S004459672006000300018

Freitas, F. L., Sparovek, G., Berndes, G., Persson, U. M., Englund, O., Barretto, A., \& Mörtberg, U (2018). Potential increase of legal deforestation in Brazilian Amazon after Forest Act revision. Nature Sustainability, 1 (11): 665-670. https://doi.org/10.1038/s41893-018-0171-4

Hartshorn, G. S. (1980). Neotropical forest dynamics. Biotropica, 12 (1): 23-30. https://doi.org/10.2307/2388152

IBGE. Instituto Brasileiro de Geografia e Estatística (2012). Manuais técnicos em geociências. Divulga os procedimentos metodológicos utilizados nos estudos e pesquisas de geociências. Rio de Janeiro, RJ, Brasil.

IBGE. Instituto Brasileiro de Geografia e Estatística (2017). Área territorial, Brasil. https://www.ibge.gov.br/estatisticas-novoportal/por-cidade-estadoestatisticas.html

Marques, A. C, \& Nucci, J. C. (2007). Planejamento, gestão e plano de manejo em unidades de conservação. Revista Ensino e Pesquisa, 4: 33-39.

Nascimento, J. L. A. (2009). Uso de geotecnologia no monitoramento de unidades de conservação: ocupações peri urbanas na Apa margem esquerda do Rio Negro, Manaus. Programa de Pós-Graduação em Ciências Ambientais e Sustentabilidade na Amazônia. Universidade Federal do Amazonas, Dissertação de Mestrado, 118p.

Nogueira, E. M., Yanai, A. M., Vasconcelos, S. S., Graça, P. M. L. A., \& Fearnside, P. M (2018). Carbon stocks and losses to deforestation in protected areas in Brazilian Amazonia. Regional environmental change, 18 (1): 261-270. https://dx.doi.org/10.1007/s10113-017-1198-1

Pinheiro, E. D. S. \& Durigan, G. 2009. Dinâmica espaço-temporal (1962-2006) das fitofisionomias em unidade de conservação do Cerrado no sudeste do Brasil. Revista Brasileira de Botânica, 32(3): 441-454. https://doi.org/10.1590/S0100-84042009000300005

Piontekowski, V. J., Matricardi, E. A T., Pedlowski, M. A., \& Fernandes, L. C. (2014). Avaliação do desmatamento no Estado de Rondônia entre 2002 e 2011. Floresta e Ambiente, 21 (3): 297-306. https://doi.org/10.1590/2179-8087.068213

Rondônia. (2002). Decreto $\mathrm{n}^{\circ} 1.144$ de 12 de dezembro de 2002. Dispõe sobre o Sistema Estadual de Unidades de Conservação da Natureza de Rondônia SEUC/RO e dá outras providências.

Sathler, D., Adamo, S., \& Lima, E. (2018). Deforestation and local sustainable development in Brazilian Legal Amazonia: an exploratory analysis. Ecology and Society, 23 (2): 30. https://doi.org/10.5751/ES-10062-230230

Sedam. Secretaria de Estado do Desenvolvimento Ambiental. (2012). Boletim Climatológico de Rondônia - 2010: Estação experimental do município de Cacoal. Porto Velho, SEDAM, 34p.

Soares-Filho, B., Alencar, A., Nepstad, D., Cerqueira, G., Vera Diaz, M. D. C., Rivero, S. \& Voll, E. (2004). Simulating the response of land-cover changes to road paving and governance along a major Amazon highway: the Santarém-Cuiabá corridor. Global Change Biology, 10 (5): 745-764. https://doi.org/10.1111/j.1529-8817.2003.00769.x

Soares-Filho, B., Nepstad, D., Curran, L., Cerqueira, G., Garcia, R., Ramos, C., Voll, E., McDonald, A., Lefebvre, P., Schlesinger, P., \& McGrath, D. (2005). Cenários de desmatamento para a Amazônia. Estudos Avançados, 19 (54): 137-152. https://doi.org/10.1111/j.1529-8817.2003.00769.x

Souza, J. L., Côrte, D. A. A. \& Ferreira, L. M. (2012). Perguntas e respostas sobre reserva particular do patrimônio natural. ICMBio, 75 p.

USGS. United States Geological Survey (2018). USGS: Science for a changing world. http://earthexplorer.usgs.gov

Whitmore, T. C. (1982). On pattern and process in forests. In: The plant community as a working mechanism. EI Newman, Blackwell Scientific Publications, pp. 45-59. 